\title{
Adaptación de la Escala de Afectividad Positiva y Negativa (PANAS) a la población de adultos de la ciudad de Córdoba: análisis psicométricos preliminares*
}

\author{
Adaptation of Positive and Negative Affectivity Scale (PANAS) \\ to adults in Cordoba city: Preliminary psychometric analysis
}

Recibido: febrero 1 de 2010 | Revisado: febrero 25 de 2011 | Aceptado: julio 26 de 2011

\author{
MÓNICA MORIONDO ** \\ Patricia De Palma \\ LEONARDo AdRIÁn MEDRANO \\ Pablo Murillo \\ Universidad Nacional de Córdoba, Argentina
}

SICI: 2011-2777(201203)11:1<187:APANAS>2.0.CO;2-L

Para citar este artículo: Moriondo, M., Palma, P., Medrano, L. A. \& Murillo, P. (2012). Adaptación de la Escala de Afectividad Positiva y Negativa (PANAS) a la población de adultos de la ciudad de Córdoba: análisis psicométricos preliminares. Universitas Psychologica, 11 (1), 187-196.

Artículo de investigación en psicometría.

* Av. Haya de la Tore s/n, Pabellón Argentina, Ciudad Universitaria, Córdoba, Argentina. E-mails: monica689@hotmail.com, patriciadpalma@yahoo.com.ar, leomedpsico@gmail.com, pabloamuri1lo@gmail.com
RES U M E N

El afecto o emoción como tema de investigación científica ha despertado un gran interés en los últimos años, sin embargo, en nuestro medio no se cuenta con instrumentos psicométricos que permitan una medición válida y confiable. El presente trabajo tuvo por objetivo realizar una adaptación psicométrica de la Escala de Afectividad Positiva y Negativa ([PANAS], Watson, Clark \& Tellegen, 1988) a la población de adultos de la ciudad de Córdoba, Argentina. Los estudios de estructura interna sugieren la existencia de dos dimensiones subyacentes homogéneas $(\alpha=0.73 ; \alpha=0.82)$ que explican un $39 \%$ de la variabilidad de la prueba. Asimismo, los estudios de correlación ítem-total, de discriminación de ítem y de contrastación de grupos resultaron semejantes a los reportados por trabajos antecedentes. En conjunto, los resultados obtenidos son promisorios y avalan el uso del PANAS en el contexto cordobés.

Palabras clave autores:

Emociones positivas, emociones negativas, adaptación psicométrica, PANAS.

Palabras clave descriptores:

Pruebas psicológicas, investigación cuantitativa, psicometría.

\section{A B S T R A C T}

The affect or emotion as a topic of scientific investigation has been of great interest in recent years, however in our environment there are no psychometric instruments which allow a valid and reliable measurement. The object of this work was to create a psychometric adaptation of the Positive and Negative Affectivity Scale ([PANAS], Watson, Clark \& Tellegen, 1988) for the adult population of the city of Cordoba, Argentina. The internal structure studies suggest the existence of two subjacent homogenous dimensions ( $\alpha=0.73 ; \alpha=0.82$ ) which explain 39\% of the variability of the test. Studies of the correlation item total, of item discrimination and contrasted groups resulted similar to those reported in previous works. Altogether, the results obtained are promising and support the use of PANAS in Cordoba. Key words authors:

Positive emotions, negative emotions, psychometric validation, PANAS.

Key words plus:

Psychological tests, quantitative research, psychometry 


\section{Introducción}

Tradicionalmente, la emoción ha sido definida como un fenómeno relativamente aislado en el tiempo que experimentamos con independencia de nuestra voluntad, y que se produce con respecto a un objeto o persona concreta. Esta característica diferencia a las emociones de los estados de ánimo, los cuales se caracterizan por ser más difusos y globales y de carecer de un objeto concreto, que puede ser indefinido o extremadamente general. Sin embargo, las emociones y los estados de ánimo poseen en común un factor de gran importancia: los fenómenos afectivos. Es decir, el afecto constituye el núcleo central de las emociones, y puede ser considerado como la molécula básica de todos los fenómenos emocionales (Dols, Carrera \& Oceda, 2002). Por ello muchas autores utilizan como sinónimos y de manera intercambiable las palabras "afecto" y "emoción" (Carr, 2007; Seligman, 2003).

Sumado a lo anterior, se han desarrollado diferentes taxonomías para clasificar las emociones (Carr, 2007). Entre los modelos contemporáneos, cabe destacar el "modelo circumplejo o dimensional", según el cual las emociones pueden clasificarse sobre la base de dos dimensiones: la valencia (agradable-desagradable) y la activación (alta-baja). De esta manera habría emociones agradables de baja y alta activación (satisfecho y excitado, por ejemplo), y emociones desagradables de baja y alta activación (abatido y ansioso, por ejemplo). No obstante, estudios basados en el análisis factorial sugieren la existencia de un "modelo ortogonal de las emociones" más parsimonioso, que postula la existencia de dos factores de relativa independencia entre sí, las emociones positivas y las emociones negativas (Watson, Clark \& Tellegen, 1988).

Si bien no existe un acuerdo unánime respecto a la conceptualización de las emociones positivas, en general este constructo hace referencia a un conjunto de emociones tales como alegría, gratificación, entusiasmo y complacencia, entre otras. Aunque son fenomenológicamente distintas comparten la propiedad de ampliar los repertorios de pensamiento y de acción de las personas, permitiendo aumentar los recursos físicos, psicológicos y sociales disponibles (Vecina-Jiménez, 2006).

Las emociones positivas cumplen una serie de funciones evolutivas que complementan las funciones de las emociones negativas (Fredrickson, 2001). Mientras que las emociones negativas se ocupan de indicar los límites y peligros, y favorecer nuestra supervivencia, por su parte, las emociones positivas favorecen el crecimiento personal y la conexión social.

Aunque las emociones experimentadas por las personas constituyen un tópico de gran relevancia actual y con múltiples y variadas repercusiones en la acción profesional (Isen, 2007), son pocos los instrumentos psicométricos que permiten una medición válida y confiable de este constructo. Quizás la escala más utilizada para medir las emociones positivas y negativas sea la Escala de Afectividad Positiva y Negativa- PANAS (Watson et al., 1988). Dicho instrumento mide dos dimensiones: la Afectividad o Emocionalidad Positiva (AP) y la Afectividad o Emocionalidad Negativa (AN). Tradicionalmente, los estudios destinados a indagar la estructura subyacente de los estados afectivos, han reportado de manera reiterada la existencia de dos factores consistentes y relativamente independientes entre sí (Watson et al., 1988). Por un lado, la dimensión Afectividad Positiva, la cual refleja el grado en que una persona se siente entusiasmada, activa y alerta y, por otro, la dimensión Afectividad Negativa, que se define como una dimensión general subjetiva displacentera que involucra un amplio rango de emociones tales como miedo, enojo, congoja y nerviosismo.

Aunque la utilización de los términos positivo y negativo puede sugerir la existencia de dos factores opuestos y correlacionados inversamente entre sí, los hechos sugieren que la existencia de una estructura ortogonal e independiente entre los factores, representa mejor la relación entre ambas dimensiones de la afectividad. Vale decir, las dimensiones Positiva y Negativa son independientes entre sí, lo cual indicaría que elevadas puntuaciones en una escala no implicarían bajas puntuaciones en la otra. Sumado a ello, la literatura señala que las dimensiones AP y AN se encuentran vinculadas 
con diferentes tipos de variables. De esta manera, la AN (pero no la AP) se relaciona con altos niveles de estrés, pobre capacidad de afrontamiento y dolencias físicas. Por su parte, la AP (pero no la AN) se encuentra relacionada con una rica vida social y con altos niveles de satisfacción vital (Watson et al., 1988).

Para desarrollar el PANAS se realizaron en primera instancia estudios tendientes a identificar las dimensiones de la afectividad y clarificar su naturaleza. Para ello se partió de un amplio número de términos que describieran diferentes estados de ánimo (57-65). Posteriormente, se seleccionaron aquellos términos que eran relativamente más puros para medir cada dimensión, es decir, términos que presentaran una considerable carga factorial en una de las escalas (superior a 0.4) y muy baja saturación en la otra (inferior a 0.25). Utilizando este criterio se llegó a un pool de 12 términos para la escala de AP y de 25 para la escala de AN. Dado que los autores buscaban desarrollar una escala breve y de rápida administración, se continuaron los estudios hasta reducir el número de términos a 10 por factor. De esta manera, la versión final del PANAS cuenta con los 10 ítems por factor, que demostraron mejores propiedades psicométricas (mayor carga factorial y mayor contribución en la consistencia interna de la escala).

Una vez obtenidos los ítems finales del PANAS se realizaron una serie de estudios psicométricos, tales como análisis de la consistencia interna (obteniéndose valores alfa de Cronbach entre 0.85 y 0.89) y estudio de validez con variables externas. Particularmente, estos últimos estudios mostraron la eficacia del PANAS, para diferenciar personas con niveles elevados de ansiedad, depresión y otros síntomas psicológicos generales.

Una de las primeras adaptaciones realizadas de la escala PANAS a la población de habla española, fue la desarrollada por Sadín et al. (1999). Mediante el uso del Análisis Factorial Exploratorio (método de extracción de componentes principales), los autores determinaron la existencia de dos factores perfectamente definidos, tanto para el grupo de varones como para el de mujeres. La proporción de varianza explicada fue similar para ambos factores, en efecto, el Factor 1, Emociones Negativas, explicó el $25.5 \%$ de la variabilidad de los ítems de la escala, mientras que el Factor 2, Emociones Positivas explicó el 23.7 \%. Sumado a esto, el Análisis Factorial Confirmatorio verificó la estructura bifactorial de la escala y su ajuste a los datos $(\mathrm{NFI}=0.89$; $\mathrm{CFI}=0.91$;FI $=0.91$; $\mathrm{RMSEA}=0.04)$.

Otro estudio de adaptación del PANAS fue realizado por Robles y Páez (2003) para la población mexicana. De la misma manera que en la adaptación española, los resultados del análisis factorial exploratorio sugirieron la existencia de una estructura bidimensional. En los estudios de consistencia interna se observó un valor $\alpha=0.9$ para la escala de afecto positivo y un $\alpha=0.85$ para la escala de afecto negativo. Por otra parte, los estudios de estabilidad realizados mediante el procedimiento test-retest fueron insatisfactorios, dado que se obtuvieron correlaciones inferiores a $r=0.4$. Sin embargo, dichos resultados son esperables a nivel teórico, ya que las emociones constituyen un constructo sumamente lábil y modificable (Casullo, 2002).

Aunque la escala PANAS, constituye uno de los instrumentos más utilizados en el mundo para medir las emociones positivas y negativas de las personas (Carr, 2007), esta no se encuentra adaptada a la población cordobesa, por lo que no resulta recomendable su utilización. En efecto, como señala Fernández (2008), el uso de un test en un contexto cultural diferente al original genera grandes dificultades. Más allá del idioma, la familiaridad con los estímulos del test, las características de la muestra de estandarización y el funcionamiento de los ítems son algunos ejemplos de fuentes de sesgo que pueden derivar en una incorrecta medición del constructo de interés. Por esto, resulta indispensable llevar a cabo estudios de adaptación, con el objeto de evaluar la equivalencia del instrumento. Tomando esto en consideración, el presente trabajo tiene por objetivo realizar una adaptación de la escala PANAS a la población de adultos de la ciudad de Córdoba. 


\section{Metodología}

\section{Participantes}

Participaron un total de 205 adultos con edades comprendidas entre los 25 y 65 años $(M=38.1$; $D E=11.7)$, que residían en la ciudad de Córdoba y que fueron seleccionados mediante un muestreo no probabilístico accidental. El $42.9 \%$ de la muestra estaba conformado por hombres y el restante $57.1 \%$, por mujeres. Respecto al nivel de estudio, se observó que una pequeña proporción de la muestra (4.4\%) contaba solo con estudios primarios, mientras que la mayor proporción (31.4 \%) había realizado estudios secundarios. La muestra contó además con participantes con estudios terciarios (14.2\%), universitario incompleto (27\%) y universitario completo $(23 \%)$. La mayor parte de los participantes eran profesionales independientes $(29.8 \%)$ y empleados en relación de dependencia (24.9\%). Las restantes ocupaciones observadas fueron: estudiantes universitarios $(23.4 \%)$, comerciantes (10.7\%), docentes (10.2 \%) y amas de casa (7.2\%). Solo un $1 \%$ de la muestra se encontraba sin empleo.

Cabe señalar que ninguno de los participantes consumía antidepresivos u otro tipo de fármaco que pudiera distorsionar o sesgar las respuestas. Finalmente, se debe destacar que el tamaño de la muestra resulta acorde con los análisis estadísticos planificados, ya que se cuenta con 10 participantes por ítem. Criterio sugerido por Anastasi y Urbina (1998) a los fines de lograr resultados útiles y estables en los estudios de adaptación psicométrica.

\section{Instrumento}

Se trabajó con la versión final de la Escala de Afectividad Positiva y Negativa (PANAS) de Watson et al. (1988), la cual consta de 20 palabras que describen distintos sentimientos y emociones, y donde el evaluado debe indicar utilizando una escala de cinco posiciones en qué medida experimenta cada una de dichas emociones (activo, fuerte, inspirado, por ejemplo). Los estudios psicométricos realizados señalan que la PANAS presenta una estructura factorial clara de dos dimensiones, una elevada consistencia interna en ambas escalas (valores alfa de Cronbach entre 0.85 y 0.89).

Dado que no se han realizado estudios locales de adaptación del PANAS, no se conocen las propiedades psicométricas de este instrumento en nuestro medio. Por esto, la estructura interna del instrumento así como algunas de sus propiedades psicométricas serán examinadas en el presente estudio.

\section{Procedimiento y análisis de datos}

En primer lugar, se llevó a cabo un estudio piloto de carácter exploratorio para realizar un sondeo preliminar, en un nivel más reducido del cuestionario y de aspectos operativos, como por ejemplo la administración del mismo y la comprensión de los ítems; posteriormente, se llevó a cabo la administración de la escala. Cabe destacar que los participantes aceptaron ser parte del estudio de manera voluntaria y brindando su consentimiento informado. Los datos recabados fueron cargados y analizados en el paquete SPSS 11.5.

Antes de llevar a cabo los análisis estadísticos planificados, se realizó un análisis de exploración inicial de datos tendiente a verificar el cumplimiento de los supuestos estadísticos y evaluar la existencia de valores extremos que pudieran sesgar los análisis. A continuación, para observar si se mantenía la estructura factorial de la escala original, se realizó un Análisis Factorial Exploratorio. Adicionalmente, se realizó un análisis de cada reactivo y se estimaron dos coeficientes alfa de Cronbach para evaluar la consistencia interna de cada escala.

Por último, se llevaron a cabo estudios tendientes a evaluar la existencia de diferencias según grupos contrastados de acuerdo al género, la edad y el nivel de estudios, para ello se utilizó la prueba $t$ de Student para muestras independientes, el coeficiente de correlación de Pearson y la prueba de Análisis de Varianza de una vía, respectivamente. 


\section{Resultados}

\section{Adaptación preliminar: estudio piloto y evaluación del proceso de respuesta.}

Tal como sugiere Grasso (2006), antes de aplicar el cuestionario a la muestra definitiva se realizó un estudio de carácter exploratorio a escala reducida, tendiente a examinar el funcionamiento del cuestionario. Con este objetivo se llevaron a cabo dos entrevistas focales (focus group) a un total de 19 participantes a quienes se les solicitó su opinión acerca de la comprensión del instrumento y el modo en que respondían a cada ítem. Las sesiones tuvieron una duración aproximada de 40 minutos. Mientras uno de los miembros del equipo mantenía moderaba discusión, otro de los miembros registraba en una lista de chequeo las evaluaciones que los participantes efectuaban sobre los siguientes aspectos formales del instrumento: 1) comprensión de los ítems, 2) comprensión de las opciones de respuesta, 3) comprensión de la codificación, 4) longitud del instrumento, 5) lenguaje y vocabulario utilizado, 6) ubicación de las preguntas y 7) facilidad y motivación para responder.

En general, no se observaron problemas de comprensión respecto a la consigna, los ítems o el modo de codificación de las respuestas. Solo se observaron algunas dificultades para diferenciar entre emociones similares, como por ejemplo, "intranquilo" y "nervioso"; "atento" y "alerta”; "asustado" y "temeroso". No se manifestaron quejas sobre la longitud de la escala y la ubicación de las respuestas. La mayor parte de los participantes comunicó que se trataba de un instrumento fácil e interesante.

Finalmente, cabe señalar que en las mismas entrevistas se realzaron preguntas tendientes a analizar los procesos cognitivos comprometidos en el proceso de respuesta. El análisis cualitativo de las respuestas indicó que una proporción de los participantes pensaban en situaciones concretas en donde habían experimentado las emociones evaluadas, y en función de la frecuencia con que ocurría cada situación estimaban la frecuencia con que sentían cada emoción. No obstante, la mayor parte de los participantes se centró en identificar primero la emoción que estaba siendo evaluada y luego recordar la frecuencia con que la percibían en su vida cotidiana.

\section{Análisis de exploración inicial de datos}

$\mathrm{Al}$ analizar el patrón de datos ausentes, no se observaron variables con más del $5 \%$ de valores perdidos. Solo se observaron 4 casos atípicos univariados (valores fuera del rango \pm 3 ) en los ítems "entusiasmo" y 1 caso en el ítem "avergonzado". Se optó por no eliminarlos siguiendo las recomendaciones de Hair, Anderson, Tatham y Black (1999), quienes señalan que hacerlo cuando estos no generan distorsiones en los análisis estadísticos, llevaría a una disminución innecesaria de la representatividad de la muestra. Considerando que aun trabajando con datos univariados se pueden generar combinaciones atípicas, se realizó el procedimiento estadístico de la distancia de Mahalanobis $\left(\mathrm{D}^{2}\right)$ para la búsqueda de casos atípicos multivariados. La significación estadística se estableció mediante la distribución $t$; ningún caso superó el umbral establecido de 0.001 para la designación como caso atípico.

A continuación, se obtuvieron las medias y desviaciones estándar de las variables y se procedió a calcular los índices de asimetría y curtosis de cada una. Todas las variables presentaron índices cercanos a la normalidad (valores inferiores a \pm 1.5 ), considerando el criterio propuesto por George y $\mathrm{Ma}$ llery (2001). Se tuvieron en cuenta estos resultados al momento de seleccionar el método de extracción de factores, tal como recomiendan Costello y Osborne (2005).

Se realizó una inspección visual de los diagramas de dispersión entre pares de variables, para evaluar la existencia de relaciones lineales entre todas las variables analizadas. Finalmente, se efectuó un diagnóstico de multicolinealidad entre los ítems, con el objeto de identificar la existencia de variables altamente correlacionadas o redundantes. Aunque la técnica de análisis factorial exige un grado de intercorrelación entre los ítems, si la correlaciones inter-ítem son superiores o iguales a 0.9, es probable que se debilite el análisis y se obtenga una solución factorial final poco estable (Martínez 
Arias, 1999). No se observaron correlaciones superiores a $r= \pm 0.9$ entre ninguna de las variables.

\section{Evidencia de estructura interna: análisis factorial del PANAS}

Para analizar la estructura interna de la escala, se realizó un primer análisis tendiente a evaluar la factibilidad del análisis factorial exploratorio (AFE). Para ello se utilizó el índice de adecuación muestral KMO (Kaiser-Meyer-Olkin), el cual presentó un valor de 0.8, y la prueba de esfericidad de Bartlett que mostró resultados significativos $\left(\chi^{2}=1179.6 ; g l\right.$ $=196 ; p<0.001)$, indicando una intercorrelación adecuada para realizar el AFE.

Dado que las variables presentan índices de asimetría y curtosis cercanos a una distribución normal, se optó por seguir las recomendaciones de Costello y Osborne (2005), quienes sugieren utilizar como método de extracción el Método de Máxima Probabilidad (también denominado Máxima Verosimilitud), considerado como el método más utilizado, eficiente y no sesgado, cuando las variables poseen una distribución normal (Byrne, 2001).

Utilizando la regla Kaiser-Gutman de autovalores superiores a 1 , se obtuvieron 5 factores que explicaban un $57 \%$ de la varianza total de la prueba. No obstante, considerando que este método tiende a sobreestimar la cantidad de factores subyacentes, se optó por interpretar el gráfico scree. Este gráfico constituye el criterio de extracción de factores más empleado en la actualidad (Cohen \& Swerdlik, 2006). Se observaron dos factores antes de la pendiente, por lo cual se optó por realizar nuevamente un análisis factorial de los ítems, pero solicitando la extracción de dos factores.

De esta manera, se extrajeron dos factores que explicaban el $39.1 \%$ de la variabilidad de la prueba. Dado que los factores no mostraban correlaciones superiores a 0.35 , se optó por realizar una rotación varimax ortogonal. Los resultados obtenidos se presentan en la Tabla 1.
TABLA 1

Saturación factorial de los reactivos del PANAS (matriz rotada)

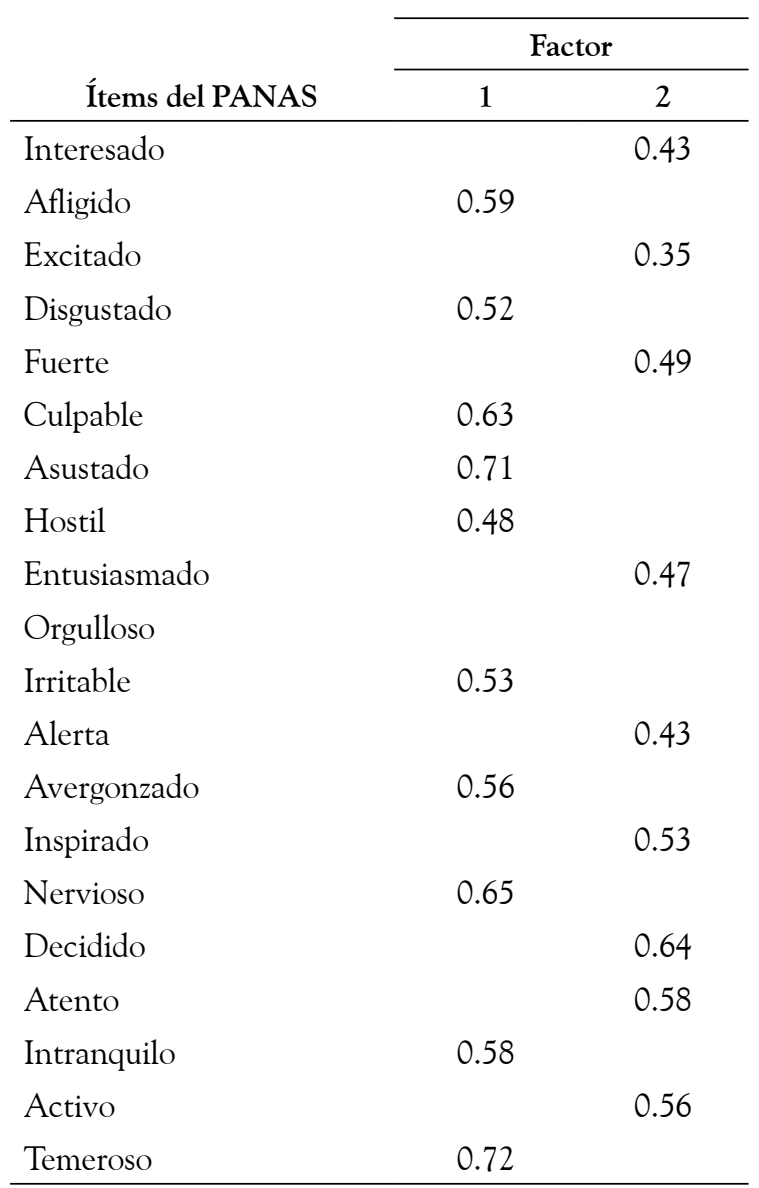

Nota. Solo se presentan las saturaciones factoriales superiores a 0.3.

Fuente: elaboración propia

\section{Análisis de ítems y consistencia interna}

Se aplicaron los procedimientos tradicionales de análisis de reactivos derivados de la teoría clásica de los tests, específicamente se calculó la correlación ítem-total y el índice de discriminación (índices D) de cada ítem (Tabla 2). Con el fin de estimar la consistencia interna de la escala, se calculó un coeficiente alfa para cada escala, observándose valores de 0.73 para la escala de afecto positivo y de 0.82 para la escala de afecto negativo. 
TABLA 2

Índices obtenidos del análisis de reactivos

\begin{tabular}{lcc}
\hline \multicolumn{1}{c}{ Ítem } & Índices D & r Ítem-Total \\
\hline Interesado & $65 \%$ & $0.56^{*}$ \\
Excitado & $43 \%$ & $0.43^{*}$ \\
Fuerte & $44 \%$ & $0.54^{*}$ \\
Entusiasmado & $60 \%$ & $0.56^{*}$ \\
Orgulloso & $12 \%$ & $0.23^{*}$ \\
Alerta & $51 \%$ & $0.5^{*}$ \\
Inspirado & $76 \%$ & $0.63^{*}$ \\
Decidido & $71 \%$ & $0.69^{*}$ \\
Atento & $53 \%$ & $0.63^{*}$ \\
Activo & $51 \%$ & $0.62^{*}$ \\
\hline
\end{tabular}

$* p<0.05$

Fuente: elaboración propia.

Diferencias entre grupos contrastados según género, nivel de estudios y edad

Se realizó una prueba $t$ para muestras independientes, comparando las puntuaciones obtenidas por hombres $(N=86)$ y mujeres $(N=113)$. Luego de verificar el supuesto de homogeneidad de la varianza mediante el test de Levene en la escala de afecto positivo $(F=3.11 ; p>0.07)$ y la escala de afecto negativo $(F=0.56 ; p>0.45)$, se calcularon los estadísticos $t$, para determinar la existencia de diferencias significativas entre los grupos. Los resultados obtenidos fueron significativos estadísticamente solo para la escala de afecto positivo $(t=2.23 ; g l=197 ; p<0.05)$, donde los hombres mostraron valores superiores $(M=33)$ a los de las mujeres $(M=31.2)$. No se observaron diferencias significativas en la escala de afecto negativo.

Respecto al nivel de estudio, se realizaron dos análisis de varianza de una vía, con el objeto de examinar la existencia de diferencias en los niveles de emociones positivas y negativas, según el nivel de estudios. Para ello, se dividió a los participantes en 5 grupos: primario completo $(N=9)$, secundario completo $(N=60)$, terciario completo $(N=29)$, universitario incompleto $(N=53)$ y universitario completo $(N=43)$. Se observaron diferencias significativas solo en la escala de afecto negativo

\begin{tabular}{lcc}
\hline \multicolumn{1}{c}{ Ítem } & Índices D & r Ítem-Total \\
\hline Afligido & $51 \%$ & $0.65^{*}$ \\
Disgustado & $28 \%$ & $0.61^{*}$ \\
Culpable & $40 \%$ & $0.69^{*}$ \\
Asustado & $40 \%$ & $0.74^{*}$ \\
Hostil & $24 \%$ & $0.55^{*}$ \\
Irritable & $50 \%$ & $0.62^{*}$ \\
Avergonzado & $50 \%$ & $0.6^{*}$ \\
Nervioso & $68 \%$ & $0.69^{*}$ \\
Intranquilo & $50 \%$ & $0.63^{*}$ \\
Temeroso & $42 \%$ & $0.73^{*}$ \\
\hline
\end{tabular}

$(F=3.16 ; p>0.01)$. Al realizar comparaciones múltiples utilizando el estadístico post hoc LSD de Fisher, se observó que los participantes de nivel de estudio primario presentaban diferencias significativas respecto al resto de los participantes. No se observaron diferencias significativas entre los restantes niveles de estudio.

Finalmente, se llevaron a cabo estudios tendientes a examinar la existencia de una asociación entre la edad de los participantes y los niveles de emociones positivas y negativas. Los resultados obtenidos sugieren la existencia de una relación significativa y con dirección negativa entre las variables edad y emociones positivas $(r=-0.20 ; p<0.05)$. Por el contrario, la relación entre edad y emociones negativas no resultó significativa.

\section{Discusión}

Si bien a nivel internacional se ha incrementado la realización de estudios empíricos destinados a examinar el rol de las emociones en el comportamiento de las personas (Casullo, 2002), en nuestro contexto son pocos los desarrollos orientados en dicho sentido. En efecto, se observa un vacío conceptual sobre la variable emociones, producto de la falta de estudios empíricos destinados a examinar la relación existente entre dicha variable y otras 
variables de relevancia, como por ejemplo el bienestar psicológico. Quizás la falta de instrumentos psicométricos debidamente adaptados a nuestro medio, constituya una de las principales razones por las que no se conocen estudios locales sobre el tópico de las emociones positivas y negativas.

Tomando esto en consideración, resulta de importancia desarrollar estudios de adaptación que cumplan con la normativa psicométrica internacional y que permitan medir de manera válida y confiable las emociones positivas y negativas de la población adulta de Córdoba. Con este objetivo se inició la adaptación de la escala PANAS (Watson et al., 1988). Los resultados obtenidos son similares a los observados en estudios antecedentes (Sandín et al, 1999), de hecho se observó que el PANAS mantiene su estructura teórica de dos factores. Con excepción del ítem "orgulloso", todos los ítems de la escala PANAS presentaron una saturación factorial coherente y con claro significado teórico.

Se considera que la baja correlación observada del ítem "orgulloso" con ambos factores del PANAS proviene de sesgos culturales que podrían afectar el comportamiento del ítem. Tal como señala Góngora (2009), este mismo concepto puede asociarse a una visión general de sí mismo como valioso (autoestima positiva, por ejemplo) o también puede asociarse a sentimientos de superioridad, egoísmo, arrogancia y narcisismo. Quizás por ello, el ítem "orgulloso" presenta correlaciones similares en la escala de emociones positivas y en la escala de emociones negativas. Una alternativa viable para solucionar esta dificultad, consistiría en diferenciar entre "estar orgulloso" y "ser orgulloso". En efecto, los estudios realizados por Robles y Páez (2003) demostraron que la conceptualización del orgullo como un rasgo, se asocia a emociones negativas (soy orgulloso), mientras que la conceptualización del orgullo como un estado, se asocia a emociones positivas (me siento o estoy orgulloso).

Otro ítem que presentó una baja saturación factorial fue "excitado". Nuevamente, se considera que la significación diferencial del mismo puede deberse a factores culturales. En efecto, las personas pueden interpretar este concepto como un estado emocional caracterizado por la motivación o interés para realizar una tarea determinada, o bien asociarlo a un estado de excitación sexual. Esta doble significación fue observada en algunos comentarios de los participantes del focus group, y también ha sido reportada por Robles y Páez (2003), quienes optaron por reemplazar la palabra "excitado" por "emocionado". Sin embargo, debe considerarse que, si bien la saturación fue baja (0.35), esta no resulta inferior a las exigidas por la normativa internacional (APA, 1999). Sumado a ello, el ítem "excitado" presentó una correlación elevada con el total de la escala, asî como una adecuada discriminación entre personas con alta y baja emocionalidad positiva.

Sintetizando, a pesar de que el ítem "orgulloso" no funcionó como se hipotetizaba, los restantes ítems se agruparon en dos factores subyacentes que explicaban más del $39 \%$ de la varianza de los ítems y que resultaban coherentes con estudios antecedentes, así como con la estructura teórica formulada por Watson et al. (1988). Esto sugiere que, en términos generales, la escala PANAS cuenta con evidencias satisfactorias de estructura interna.

El examen de la consistencia interna del instrumento resultó aceptable, dado que el coeficiente alfa de Cronbach presentó un valor 0.73 para la escala de afecto positivo y de 0.82 para la escala de afecto negativo. Esto sugiere que ambas escalas muestran un grado adecuado de homogeneidad entre los ítems, lo cual aumenta la precisión del instrumento (Cohen \& Swerdlik, 2006). No obstante, sería recomendable desarrollar nuevos estudios tendientes a aumentar la consistencia de la escala de emociones positivas. En efecto, debería chequearse empíricamente si la escala se vuelve más consistente cambiando los ítems "orgulloso" y "excitado" por "estar orgulloso" y "emocionado", respectivamente.

Finalmente, respecto a los estudios tendientes a examinar la relación entre las puntuaciones obtenidas por el PANAS con variables externas, cabe señalar que, en términos generales, los resultados fueron similares a los reportados por estudios previos. En primer lugar, se observaron diferencias en los niveles de emociones negativas y el nivel de estudio. De hecho, las personas que solo contaban con estudios primarios presentaban mayores puntuaciones en las escalas de emociones negativas. 
Este tópico fue estudiado por Seligman (2003) quien sugirió que, si bien el nivel de estudio generalmente no se asocia con las emociones, sí pueden observarse altos niveles de emocionalidad negativa, en personas que cuentan con necesidades básicas insatisfechas y bajos niveles de estudio. De esta manera, los resultados obtenidos en el presente trabajo son coherentes con los reportados por Seligman, manteniéndose la hipótesis que sugiere que las personas con bajo nivel de estudio presentan mayores dificultades para satisfacer sus necesidades vitales básicas y, en consecuencia, experimentan mayores emociones negativas.

Respecto a la variable edad, los resultados también fueron similares a los comunicados por la literatura (Casullo, 2002; Seligman, 2003). En efecto, se observó una correlación negativa y significativa entre la edad y las emociones positivas. Esto sugiere que las personas más jóvenes tienden a experimentar mayores emociones positivas. Aunque no se posee una teoría clara que permita fundamentar el por qué de esta relación, los estudios empíricos desarrollados son consistentes respecto al hecho de observar que las personas más jóvenes muestran mayores "picos" de emociones positivas.

Por último, contrariamente a lo esperado, se observó que los hombres presentaban mayores niveles de emociones positivas que las mujeres. Este resultado es inverso al observado en estudios previos (Mestre, Sampur \& Tur, 2009), donde se observaba que las mujeres presentaban mayores niveles de empatía, mayores comportamientos prosociales y mayores emociones positivas que los hombres. Se deberían desarrollar nuevos estudios al respecto, con muestras más amplias y heterogéneas, para evaluar si estas diferencias se mantienen.

Entre las limitaciones del presente trabajo cabe señalar que no se verificaron algunas propiedades psicométricas importantes, como la estabilidad temporal de la escala, o su relación convergente o discriminante con otros instrumentos. De la misma manera, sería adecuado desarrollar estudios de carácter confirmatorio para verificar la estructura factorial del PANAS. Sin embargo, se debe destacar que los estudios psicométricos examinados mostraron resultados satisfactorios y alentadores, los cuales sugieren que la escala PANAS constituye un instrumento inicialmente válido y confiable, para ser utilizado en la población de adultos de Córdoba.

\section{Referencias}

American Psychological Association (1999). Standards for psychological and educational tests. Washington, D.C.: Authors.

Anastasi, A. \& Urbina, S. (1998). Test psicológicos. México: Prentice Hall.

Byrne, B. M. (2001). Structural equation modeling with AMOS: Basic concepts, applications, and programming. Mahwah, NJ: Lawrence Erlbaum.

Carr, A. (2007). Psicología positiva. La ciencia de la felicidad. Buenos Aires: Paidós.

Casullo, M. M. (2002). Evaluación del bienestar psicológico en Iberoamérica. Buenos Aires: Paidós.

Cohen, R. J. \& Swerdlik, M. E. (2006). Pruebas y evaluación psicológicas. Madrid: McGraw Hill.

Costello, A. B. \& Osborne, J. W. (2005). Best practices in Exploratory Factor Analysis: Four recommendations for getting the most from your Analysis. Practical Assessment, Research EF Evaluation, 10(7), 1-9.

Dols, J. F., Carrera, P. \& Oceja, L. (2002). Bases sociales de la emoción. En J. F. Morales (Coord.), Psicología Social (pp. 235-285). Buenos Aires: Prentice Hall.

Fernández, A. (2008). Adaptación de test a otras culturas. En S. Tornimbeni, E. Pérez \& F. Olaz (Eds.), Introducción a la psicometría (pp. 191-205). Buenos Aires: Piadós.

Fredrickson, B. L. (2001). The value of positive emotions. American Scientist, 91, 330-335.

George, D. \& Mallery, M. (2003). Using SPSS for Windows step by step: A simple guide and reference. Boston, MA: Allyn \& Bacon.

Góngora, V. (2009). La autoestima. En M. Casullo (Comp.), Prácticas en psicología positiva (pp. 81-110). Buenos Aires: Lugar Editorial.

Grasso, L. (2006). Encuestas. Elementos para su diseño y análisis. Córdoba: Encuentro Grupo Editor.

Hair, J. F., Anderson, R. E., Tatham, R. L. \& Black, W. (1999). Análisis multivariante. Madrid: Prentice Hall.

Isen, A. (2007). El afecto positivo como fuente del potencial humano. En G. Aspinwall \& U. Stau- 
dinger (Eds.), Psicología del potencial humano (pp. 247-268). Madrid: Gedisa.

Martínez Arias, M. R. (1999). El análisis multivariante en la investigación científica. Madrid: La Muralla.

Mestre, M., Sampur, P. \& Tur, A. (2009). Empatía y conducta prosocial. En M. Casullo (Comp.), Prácticas en psicología positiva (pp. 231-268). Buenos Aires: Lugar Editorial.

Robles, R. \& Páez, F. (2003). Estudio sobre la traducción al español y las propiedades psicométricas de las escalas de afecto positivo y negativo (PANAS). Salud Mental, 26(1), 69-75.

Sadín, B., Chorot, P., Lostao, L., Joiner, T. E., Santed, M. \& Valiente, R. (1999). Escalas PANAS de Afecto
Positivo y Negativo: validación factorial y convergencia transcultural. Psicothema, 11(1), 37-51.

Seligman, M. (2003). La auténtica felicidad. Madrid: Vergara.

Vázquez, C. (2006). La psicología positiva en perspectiva. Papeles del Psicólogo, 27(1), 1-2.

Vecina-Jiménez, M. L. (2006). Emociones positivas. Papeles del Psicólogo, 27(1), 9-17.

Watson, D., Clark, L. A. \& Tellegen, A. (1988). Development and validation of brief measures of positive and negative affect: The PANAS Scales. Journal of Personality and Social Psychology, 54, 1063-1070. 